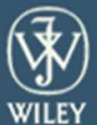

Color Research and Application

WILEY

\title{
Improving Color Reproduction Accuracy of an OLED-based Mobile Display
}

\begin{tabular}{|r|l|}
\hline Journal: & Color Research and Application \\
\hline Manuscript ID & COL-16-120.R2 \\
\hline Wiley - Manuscript type: & Research Article \\
\hline Date Submitted by the Author: & n/a \\
\hline Complete List of Authors: & $\begin{array}{l}\text { Kirchner, Eric; AkzoNobel Performance Coatings, Color Research } \\
\text { van der Lans, Ivo; AkzoNobel Performance Coatings, Color Research } \\
\text { Perales, Esther; Universidad de Alicante, Department of Optics, } \\
\text { Pharmacology and Anatomy } \\
\text { Martínez-Verdú, Francisco; University of Alicante, Optics, Pharmacology } \\
\text { and Anatomy }\end{array}$ \\
\hline Keywords: & sRGB color space, Color measurement, Display technology, Color accuracy \\
\hline &
\end{tabular}




\title{
Improving Color Reproduction Accuracy of an OLED-based Mobile Display
}

\author{
Eric Kirchner ${ }^{1 *}$, Ivo van der Lans ${ }^{1}$, Esther Perales ${ }^{2}$ and \\ Francisco Martínez-Verdú ${ }^{2}$ \\ ${ }^{1}$ Color Research department, AkzoNobel Performance Coatings, Rijksstraatweg 31, 2171 AJ Sassenheim, The Netherlands \\ 2 Color \& Vision Group, University of Alicante, Carretera de San Vicente del Raspeig s/n 03690, Alicante, Spain
}

For improving color reproduction accuracy of mobile displays, we recently developed a generic model for device-specific display characterization model that also accounts for the influence of illuminance from ambient light. In the present article, this MDCIM model (Mobile Display Characterization and Illumination Model) is applied to a Samsung Galaxy S4 display, representing OLED displays. The performance of the model was tested by determining the values of all model parameters using publicly available technical data only. We organized visual tests under various ambient illuminance levels from 600 to 3000 lux. Seven observers compared the color of displayed images with the color of physical samples. With the MDCIM method, the quality of the color match was shown to improve considerably as compared to using only deviceindependent encoding color space. On a five-point scale to quantify color reproduction accuracy, the MDCIM resulted in more than one unit improvement at 1000 lux illuminance. At lower and higher illuminance, the improvement was even larger. Color reproduction accuracy was found to be at least reasonable, according to the subjective assessment of visual observers, for more than $75 \%$ of the samples when using the MDCIM method, but only $20 \%$ or less when using the common device-independent encoding color space.

Key words: sRGB color space; Color measurement; Display technology; Color accuracy 


\section{INTRODUCTION}

After the introduction of the iPhone in 2007 and the iPad in 2010, mobile displays quickly became a major class of electronic displays. For generating colors on a mobile display, usually sRGB is used as deviceindependent color space, thereby assuming that the firmware of the mobile device maximizes color accuracy. However, there are several reasons why this assumption may not be completely correct, and indeed there is a strong momentum towards improving the color reproduction accuracy of mobile displays ${ }^{1}$. For example, manufacturers may have optimized the firmware to maximize color preference rather than color reproduction accuracy. But also the level of ambient lighting is expected to affect color reproduction accuracy. Mobile displays are typically used in a wide variety of ambient lighting conditions ${ }^{2}$. Even for indoor office applications illuminance levels vary between typically 500 and $2000 \mathrm{lux}^{3}$. As is well known, the ambient illuminance level strongly influences the optimum color representation.

For improving the color reproduction accuracy of mobile displays, we recently developed the Mobile Display Characterization and Illumination Model (MDCIM) ${ }^{4}$. It takes the following two aspects into account: (i) a display characterization model that is specific for the display, (ii) the ambient illuminance level. We already tested the performance of the MDCIM model for the Apple iPad Air 2 display, as representing a mobile LCD-based display ${ }^{4}$. In the present article we apply the MDCIM model to the color reproduction accuracy of the display of the Samsung Galaxy S4, a well-known representative for OLED displays. We will also show how our results can be extend to many other mobile displays.

For creating images with the MDCIM model, reflection values from physical samples are required as deviceindependent colorimetric input data. Therefore, the MDCIM model is not applicable for cases in which only input data is available from more common image capturing devices, such as cameras and flatbed scanners. Also for soft proofing tasks that aim at maximizing the perceived color match between displayed images and printed images $^{5}$, the MDCIM model is not suitable (unless reflection data from the printed images are available).

In the present article, we determined the accuracy of the MDCIM model for the case that only independently published technical specifications of the display are used. We made no attempt to improve its color reproduction accuracy by optimizing model parameters specifically for this test. Therefore we consider our results as an objective measure of the color reproduction accuracy of the MDCIM model. 
The relevant expressions for the display characterization model that is part of the MDCIM model are reviewed briefly in section 2a, whereas the method to account for different ambient illuminance levels and adaptation is summarized in section $2 \mathrm{~b}$. More details on the MDCIM model can be found in our previous publication ${ }^{4}$. In section 3 we describe the experimental details for setting MDCIM model parameters and for setting up the visual test that was performed to test the accuracy of the MDCIM model. Results are discussed in section 4, after which we wrap up with conclusions and recommendations.

\section{THE MDCIM MODEL}

\section{2a Display Characterization Model}

The default device-independent color space is sRGB color space. It is based on the Gain-Offset-Gamma model from Berns ${ }^{6,7}$, which relates the tristimulus parameters $X, Y, Z$ (which in turn are linked to colorimetric parameters such as CIELAB) to the luminances $Y_{R}, Y_{G}$ and $Y_{B}$ of the red, green and blue channel of a CRT display:

$$
\left(\begin{array}{c}
X \\
Y \\
Z
\end{array}\right)=M\left(\begin{array}{l}
Y_{R} \\
Y_{G} \\
Y_{B}
\end{array}\right)
$$

Here, it is assumed that the luminances of the red, green and blue channel show an exponential dependence on the digital value $d_{R}, d_{G}$ and $d_{B}$ for each color channel (each scaled between 0 and 1):

$$
\begin{aligned}
& \left(\begin{array}{l}
Y_{R} \\
Y_{G} \\
Y_{B}
\end{array}\right)=\left(\begin{array}{c}
\left(k_{1, R} d_{R}+k_{2, R}\right)^{\gamma_{R}} \\
\left(k_{1, G} d_{G}+k_{2, G}\right)^{\gamma_{G}} \\
\left(k_{1, B} d_{B}+k_{2, B}\right)^{\gamma_{B}}
\end{array}\right) \text { if } d_{R}, d_{G}, d_{B}>d_{0} \\
& Y_{R}=\beta \cdot d_{R} \text { if } d_{R}<d_{0} \\
& Y_{G}=\beta \cdot d_{G} \text { if } d_{G}<d_{0} \\
& Y_{B}=\beta \cdot d_{B} \text { if } d_{B}<d_{0}
\end{aligned}
$$

The three functions on the right-hand side of equation (2) are called the Tone Rendering Curves (TRCs) or opto-electronic transfer functions. For common displays with $3 \times 8$ bits color representation, $d_{R}, d_{G}$ and $d_{B}$ are equal to the common $R G B$ values (ranging from 0 to 255 ), divided by 255 (i.e., $2^{8}-1$ ). Obviously equation (2) is not limited to displays with 8-bits color depth, but can be applied for any other digital color representation as well. 
In sRGB space, it is assumed that the matrix $M$ in equation (1) is given by the following expression ${ }^{8}$ :

$$
M_{\text {sRGB }}=\left(\begin{array}{lll}
0.4124 & 0.3576 & 0.1805 \\
0.2126 & 0.7152 & 0.0722 \\
0.0193 & 0.1192 & 0.9505
\end{array}\right)
$$

and that for all three channels the parameters gamma $\gamma=2.4$, gain $k_{1}=1 / 1.055$, offset $k_{2}=0.055 / 1.055$, linear coefficient $\beta=1 / 12.92$ and threshold $d_{0}=0.0392$ (Ref.8). As shown in the Appendix, these values can be derived from purely mathematical and pragmatic arguments, without considering the actual technical specifications of a display. The values of the matrix elements in equation (3) are related to the CIE 1931 chromaticity values $(x, y)$ and maximum luminance $Y_{\max }$ of the three channels ${ }^{9,10}$ :

$$
M=\left(\begin{array}{lll}
\frac{x_{R}}{y_{R}} Y_{R, \max } & \frac{x_{G}}{y_{G}} Y_{G, \max } & \frac{x_{B}}{y_{B}} Y_{B, \max } \\
Y_{R, \max } & Y_{G, \max } & Y_{B, \max } \\
\frac{z_{R}}{y_{R}} Y_{R, \max } & \frac{z_{G}}{y_{G}} Y_{G, \max } & \frac{z_{B}}{y_{B}} Y_{B, \max }
\end{array}\right)
$$

In this way, the numerical values for the matrix $M$ as prescribed for sRGB color space can be shown to correspond to particular values for display specifications. We note that following conventions in the cited literature, in equation (4) the total luminance of white is not scaled to unity, whereas in equation (3) this is the case. The resulting values for matrix $M$ are shown in Table 1. Therefore, the combination of equation (4) and the derivations in the Appendix together demonstrate how the values for all parameters in sRGB color space were derived for the particular set of display specifications listed in Table 1. For the display specifications, primaries were used that had already been defined in 1991 by the CCIR (Comité Consultatif International pour la Radio, now replaced by the International Telecommunication Union ITU) recommendation BT.709 for high definition television.

(Table 1)

Finally, the value of 2.4 for the gamma parameter in sRGB encoding color space is partly inherited from parametrizations dating back from before 1998, and partly based on optimizations for a reference ambient illuminance level of $64 \operatorname{lux}^{8,11}$.

Modern mobile displays are often viewed under much higher illuminance than 64 lux. The International Color Consortium recommends using different values for the gamma parameter in the case of situations 
differing from the reference viewing environment ${ }^{12}$. Modern mobile displays also have colorimetric specifications that differ considerably from those listed in Table 1. The default sRGB color space that is conveniently used as device-independent encoding color space, is therefore not expected to produce the best color reproduction color accuracy for specific displays under specific viewing conditions. Indeed, devicedependent display characterization models have already been shown to be able to improve color reproduction accuracy for non-mobile LCD displays ${ }^{13,14}$. Equations (2) and (4) represent the display characterization model that makes the MDCIM model device-dependent.

\section{$2 b$ Ambient lighting and adaptation}

In order to account for ambient illuminance one may use the CIECAM02 color appearance model. This approach was indeed used in a number of previous studies on mobile displays ${ }^{15,16,17}$. However, the CIECAM02 has been shown to be less useful for predicting the performance of mobile displays subjected to a wide variety of illumination conditions ${ }^{16,17}$. Also, even after refining the CIECAM02 model specifically for mobile displays the resulting color accuracy was shown to leave much room for improvement ${ }^{16,17}$.

For this reason, we chose a different approach to account for ambient illuminance. We noted already that the MDCIM model uses equation (4) to account for the display-specific values of maximum luminance and chromaticity coordinates. When combining the matrix elements defined in equation (4) with equation (1), tristimulus values are obtained that have the dimension of luminance. In order to calculate colorimetric parameters such as CIELAB values, the tristimulus values of reference white need to be expressed in the same dimension, by multiplying them with the luminance $L_{r w}$ of reference white:

$$
L_{r w}=M_{L} / \pi=E \rho / \pi \quad c d / m^{2}
$$

where $M_{L}$ is the luminous exitance of reference white, and $E$ is the ambient illuminance (lux) ${ }^{18}$. We assume that the reference white has an ideal reflectivity $\rho=1.0$ independent of wavelength, and that it is a perfectly diffuse Lambertian scatterer. In the MDCIM model, the level of ambient illuminance is accounted for by Equation (5).

It is also important to determine the adaptation state of the observers participating in the visual tests that are described in section 3c. Earlier tests of the MDCIM model have shown that the best color reproduction accuracy was obtained by assuming that the observers were completely adapted to the ambient lighting, i.e. 
not to the display white point ${ }^{4}$. This adaptation state indicates that at common viewing distance the physical size of the display of a smartphone is small enough to not significantly affect the adaptation state of the observer. Also, during the visual tests observers continuously change the field of view from the display to the physical samples, which results in relatively short time periods of viewing the display. Both aspects are typical for common usage of smartphones and other mobile displays, but do not apply for viewing desktop computer and television displays. Therefore results from the present analysis are considered only valid for mobile displays.

\section{EXPERIMENTAL}

\section{3a. Display characterization parameters}

Equation (4) shows some of the parameters that are needed to make the MDCIM model device specific: maximum luminance values and CIE 1931 chromaticity coordinate values for the three color channels. It is possible to measure these properties directly on the display, as we did for the Apple iPad Air 2 device ${ }^{4}$. But for the Samsung Galaxy S4 device we preferred to use only technical specifications found in documentation provided by display manufacturers or independent organizations ${ }^{19,20,21,22}$. Table 2 shows the parameter values for the Samsung Galaxy S4 smartphone as reported in Ref.23.

(Table 2)

Substituting the parameter values from Table 2 into equation (4), we obtain the following conversion matrix from $R G B$ to tristimulus $X Y Z$ values, specifically for the Samsung Galaxy S4 in Movie Mode:

$M_{\text {S4, Movie Mode }}=\left(\begin{array}{lll}1.3283 & 0.9781 & 0.6739 \\ 0.7939 & 2.1792 & 0.3674 \\ 0.2143 & 0.3763 & 2.9091\end{array}\right)$

The differences with the corresponding values (equation 3) from sRGB color space are obvious.

While almost all smartphones and tablets make it impossible for the user to change the display color settings other than by changing display luminance (which is called display brightness by most manufacturers), the Samsung Galaxy S4 (and later models of the same product line) offer a basic form of color management. Through the Settings and Display menu, the user can choose between four different screen modes. The Movie Mode that we just discussed corresponds most closely to the default sRGB parameter settings, making 
it the preferred mode when displaying independent content such as movies. But when changed to Standard Mode, the full range of colors is addressed that can be reached by current OLED technology. In this way, a color gamut is obtained that is 32 percent larger than the default gamut covered by sRGB color space ${ }^{24}$. This makes it technically possible to accurately display for example images taken with professional digital cameras that utilize Adobe RGB space. Also for applications such as electronic color documentation, the advantage of the wider color gamut is obvious, as long as good color accuracy can be ensured.

For the Standard Mode, the technical specifications as reported in Ref.25 are summarized in Table 3. When comparing these numbers with those from Table 1, it is clear that the color gamut is indeed widened considerably. This is illustrated schematically in Figure 1.

(Table 3)

(Figure 1)

The wide color gamut of the Samsung Galaxy S4 shown in Figure 1, and the very low luminance it produces when displaying a black image, are typical characteristics of OLED display technology ${ }^{26,27,28}$. A main characteristic of the LCD technology that is used in e.g. iPhones and iPads is their large maximum display luminance ${ }^{26}$.

With the technical specifications from Table 3, equation (4) produces the following conversion matrix specifically for the Standard Mode of the Samsung Galaxy S4:

$M_{\text {S4, Standard Mode }}=\left(\begin{array}{lll}1.7580 & 0.8375 & 0.6815 \\ 1.0084 & 2.3679 & 0.2825 \\ 0.2783 & 0.2768 & 3.4499\end{array}\right)$

In order to develop the MDCIM model specifically for the Samsung Galaxy S4, we also need to find best parameter values for the Tone Rendering Curves in equation (2). Also here we use data from published technical specifications ${ }^{29}$, as shown in Figure 2.

For the Samsung Galaxy S4 the GOG model gives a good fit of the measured Tone Rendering Curve, and it was found to be not necessary to use more advanced fit functions ${ }^{30,31,32,33}$. Figure 2 shows that for the Samsung Galaxy S4 the measurement data are represented well by using $\gamma=2.7$. The figure shows that this 
gives a much better representation of the measurement data than when using the default value of $\gamma=2.4$ that is specified in the device-independent sRGB model.

We note also that according to the measurement data, the Tone Rendering Curve does not change for the different display modes of the device, but obviously we could have incorporated that if this would have been necessary.

(Figure 2)

The available data do not make it possible to improve the part of the Tone Rendering Curve for $R G B$ values smaller than 10, so in that area we will keep the default values from the sRGB definitions. Equations (A5), (A6) and (A7) from the Appendix then allow us to calculate values for all required parameters: $d_{0}=10 / 255$, $k_{1}=1 / 1.0666, k_{2}=0.0666 / 1.0666$ and $\beta=1 / 20.050$.

\section{$3 b$ Visual test software}

A visual test was designed using software that was developed dedicated for this test, and which runs on mobile devices. Representative screen shots of this software are shown in Figure 3.

(Figure 3)

In the software, the user first inputs his/her name, and the illuminance level of the ambient lighting (in lux), as measured with a lux meter that is positioned close to the display. After this stage, the actual visual test starts with a screen asking the observer to pick a physical sample (Figure 3a). For every observer, the software determines a fully randomized order of samples. After the observer has placed the physical sample next to the display, a button is clicked and a new screen is shown (Figure 3b). Now, two different representations of the sample are shown in the form of colored images, surrounded by a constant white color. The top and the bottom image are calculated using the common device-independent method (that involves sRGB encoding color space) and the device-specific MDCIM model. For each sample the software randomizes which method is used for which image. The observer, who is not aware which image corresponds to which method, inputs which of the two images is perceived to be most similar in color to the physical 
sample. In the next screen, the observer is asked to input a visual score representing the perceived quality of the color match between the physical sample and the image that was preferred in the previous step (Figure $3 c)$.

Visual scores can be inputted ranging from 0 to 5 , with step size 0.25 . The descriptions for the scores are shown on the display, as reproduced in Table 4 . A score of 0 refers to the situation where no or hardly any color difference can be seen between the physical sample and the displayed image. The perceived quality of the color match gradually becomes worse for larger values of the visual score. A score of 3 indicates that the perceived color difference becomes doubtful, and at a value of 4 the match is judged to be not correct.

In the next screen, a similar screen as in Figure $3 \mathrm{c}$ is shown, allowing the observer to input a visual score for the other, not-preferred image. Finally, a verification screen is shown that asks the observer to confirm the visual scores that were inputted, or to repeat the scoring for the selected color.

The whole process is repeated for all 35 colors in the set. For each observer and each sample, three assessments are thus collected: an evaluation if the common device-independent method or the MDCIM model produced the best matching image, and a visual score for each of these two methods. When the visual tests were finished, all data was processed electronically.

(Table 4).

\section{$3 c$ Visual set-up}

Visual tests were organized at illuminance levels of 600, 1000, 1500 and 3000 lux, in a randomized order that was different for each observer. Since we tested the mobile display separately for two different display settings, Movie Mode and Standard Mode, a total of eight test sessions was needed for each observer. Seven observers (5 male, 2 female, age between 35 and 55) participated in the visual experiments. All observers have normal color vision as confirmed by the Ishihara color vision test and the Farnsworth-Munsell 100 hue test, and all were well experienced in visual testing of color differences. A total of 1960 visual assessments were collected in this way.

Observers were asked to take place behind a table inside a room that allowed setting a wide range of illumination levels. The room has no windows, which allowed us to better control the lighting inside the 
room (Figure 4a). The light spectrum in this room represents day light (D65 spectrum). In order to avoid the disturbing effect of surface gloss on visual assessments as much as possible, the lighting in the room was made as diffuse as possible. More details on this "diffuse room" can be found in Ref.34.

In order to control the observation conditions further, the observers were instructed to put the electronic display flat on the table, which was covered by neutral grey cloth. Every physical sample was held next to the display, allowing a direct visual comparison between the color shown on the display and the color of the physical sample. The display was set at maximum luminance level. In the instructions, it was also explained that a slight rocking motion ("wobble") of the physical sample helps to recognize the actual color of the sample. The observers were asked to assess the quality of the color match between physical sample and displayed image, as when the image would be used to indicate the color of a wall paint to a consumer.

Observers were also asked to view the electronic display from a straight angle (Figure 4b). This is important, because smartphones and tablets have a considerable variation of color on viewing angle. This variation results mainly in luminance shifts for LCD displays, and in shifts of chromaticity coordinates for OLED displays $^{2}$.

(Figure 4)

\section{3d. Paint samples and color collection}

In order to avoid fatigue during visual test sessions, the number of color samples needed to be limited. Therefore we selected 35 colors to represent color space. We made sure that exactly four samples were included for each of the six chromatic color categories: yellow, orange, red, purple, blue and green. For each color category, both lighter and darker colors were included, and both high and low saturation.

We decided to include eleven samples in the final category of achromatic colors. The reason to use more samples for the achromatic color category than for the chromatic color categories is because in preparing the main test it was found that the accuracy of color visualization is particularly challenging for achromatic colors, a result in line with a previous study ${ }^{9}$. 
For the physical samples we used samples from the RAL Classic collection, as developed by the German Institute for Quality Assurance and Labeling (RAL) ${ }^{35,36}$. We decided to use the RAL840HR collection because of its relatively low gloss level, which makes color evaluation easier for observers.

The list of RAL colors thus found is reproduced in Table 5. The corresponding color coordinates are graphically represented in Fig. 5. These values are all based on reflection data obtained with a multi-angle spectrophotometer with $10 \mathrm{~nm}$ resolution (BYK-Mac from BYK-Gardner). We used data obtained at the $110^{\circ}$ aspecular angle. By using this geometry, far away from the specular (mirror) angle, the influence of direct reflection from the samples is minimized. In our experience, during visual assessments of color, observers tend to assess color properties by mentally discounting for gloss contributions as well. Our choice for a measurement geometry that minimizes the influence of gloss is based on this experience.

(Table 5)

(Figure 5)

\section{3e. Gamut mapping}

Any method that aims at representing colors on a display needs to deal also with cases in which the calculated values of the $R, G$ and $B$ parameters fall outside the range that a display can handle. This problem, which is usually referred to as color representations being out-of-gamut, occurs for six from the 35 selected RAL colors when using the common device-independent method: RAL2000, 2004, 5002, 5010, 6026 and 6029. In these cases, we simply minimized the R, G and B values to 0 and maximized them to 255 for an eight-bit display. The results from the visual experiments described in this article shows that the perceived color accuracy for the common device-independent method for these colors is not worse than for the other colors. Therefore we conclude that for the common device-independent method, the simple minimization / maximization method is good enough.

For the MDCIM model we may select one or more of the many gamut-mapping methods that have been proposed in the past ${ }^{37,38}$. We limited ourselves to two relatively simple techniques, that both use CIE-Lab color space. The PHL-mapping technique preserves hue angle and lightness, by only reducing the chroma 
parameter until calculated $R G B$ values are all in the required numerical range. This technique led to good results in the past ${ }^{39}$. In the few cases where no solution could be found in this way, a second step is necessary. The PHC-mapping technique is then used, which keeps the hue angle and chromaticity values constant, while the lightness value is increased or decreased until the color is no longer out-of-gamut.

For creating color representations at 600 and 1000 lux illuminance, the MDCIM model calculated ten from the 35 RAL colors to be out-of-gamut. These include the same six colors already mentioned for the common device-independent method, with RAL 2001, 2008, 3003 and 4004 being added. For all ten colors, the PHL technique was successful.

The MDCIM model calculations for the case of 1500 lux illuminance showed four more colors to be out-ofgamut. RAL 3015 was solved by the PHL technique as well. The other three colors are those with the highest lightness values in the set: they are the only samples with $L * 85$. For these three cases it is obvious that reducing chromaticity does not bring the color representation inside the gamut, because the main problem is not color saturation but luminance (lightness). For RAL 1013 and 9010 the lightness reduction step of the PHC technique was able to bring the $R, G$ and $B$ values within range. For RAL 1016 no solution was found with the simple clipping algorithms. Instead of proposing more advanced clipping methods, we decided that in cases in which no solution is found, the software falls back to the common device-independent representation. These cases will be left out from further analysis.

In case of 3000 lux, the MDCIM model produces $R, G$ and $B$ values that are within the required range for only 9 from the 35 colors. For the remaining 26 colors we preferred not to use the clipping algorithm, since this would make it a test of the clipping method rather than an evaluation of the MDCIM model. Therefore we used a different approach. For each of these 26 colors, we calculated if the MDCIM model produces $R, G$ and $B$ values that are within the $0-255$ range at a certain cut-off value $E_{\text {cut-off }}$ for the illuminance. We chose a value $E_{\text {cut-off }}=1550$ lux, motivated by the fact that at this value for illuminance an achromatic color with lightness value $L^{*}=80$ is already predicted to become out-of-gamut when using the MDCIM model. For 12 from the 26 problematic colors, the colors were no longer out-of-gamut when using this method. For the remaining 14 colors we decided that no solution was found, and like before we used the common deviceindependent representation in the test. Also these cases are excluded from further analysis. 


\section{RESULTS}

\section{4a. Repeatability (Movie Mode)}

In order to quantify the repeatability of the visual assessments, we decided to let four observers repeat a randomly selected test session. In this way, we collected repeatability data for 14 percent of all assessments, for all three types of visual data collected in the experiment. During the repeated visual session, observers were not aware that it was a repeat session.

For the evaluation if the common device-independent method or the MDCIM model produced the best matching image, we found that in $94 \%$ of the cases the repeated session resulted in the same preference. From this, we conclude that the preference data is highly repeatable.

For the repeatability of the visual scores, we found that the average absolute difference in score between the regular session and the repeated session is 0.51 for images produced using the common device-independent method, and 0.60 when using the MDCIM model. In the analysis of visual test results, we will focus on effects that are larger than this repeatability error.

\section{4b. Preference (Movie Mode)}

One of the three scores that is inputted by each observer for each color in the set, identifies which of the two images is found to show the closest color match to the physical RAL sample. The observers were not aware which image corresponded to the common device-independent method, and which to the MDCIM model (and this correspondence varied randomly over the samples). Only in the analysis afterwards we linked the assessments to the two methods.

In Table 6 we show the percentage of observer assessments that favored the MDCIM model images over the images generated with the common device-independent method. This Table shows that for all illuminance levels investigated in this experiment, the color representations calculated by the MDCIM model are strongly favored over those from the common device-independent method.

At 1000 lux, this percentage is lowest, which is caused by the fact that at that illuminance level the common device-independent method gives the best accuracy, as shown below. However, even then the percentage of assessments favoring the MDCIM model is still high at $75 \%$ when the display is in Movie Mode. The 
percentage increases to $83 \%$ when light becomes dim at 600 lux, and to $88 \%$ when light becomes brighter at 1500 lux. At intense lighting of 3000 lux the percentage increases even to $95 \%$. We note that the latter percentage refers only to the 21 colors for which the procedure explained in section 3 e produces $R, G$ and $B$ values that are within the 0-255 range of an eight-bit display. For the remaining 14 RAL colors in the test, this procedure does not solve the problem of out-of-gamut color representation. For those colors, the MDCIM model can offer only the representation generated by the common device-independent method.

(Table 6)

\section{4c. Accuracy of the common device-independent method (Movie Mode)}

During the visual test, observers gave a visual score to quantify to quality of color match between displayed image and physical RAL sample. The scores were defined as in Table 4. If we average the visual scores over all samples, then for the device in Movie Mode we find the average scores reproduced in Table 7.

At an illuminance of 1000 lux, the common device-independent method produces an average score of 3.5. According to the description of the scores in Table 4 the color accuracy of the common device-independent method is therefore not very good, being between "Difference visible, doubtful match" and "Difference clearly visible, not correct match”.

At 600 lux, the same average score is found. At higher illuminance levels, the average score increases to 4.1 and 4.4. This shows that the color accuracy of the common device-independent method becomes increasingly worse for 1500 and 3000 lux. This may also be quantified by calculating the percentage of samples with an average visual score of 4 ("Difference clearly visible; not correct match") or worse. When illuminance increases from $600,1000,1500$ to 3000 lux, the corresponding percentage increases from $11 \%, 31 \%$ and $77 \%$ to $84 \%$. We conclude that for a vast majority of the samples, the color accuracy is unsatisfactory at 1500 lux and above.

(Table 7)

4d. Accuracy of the MDCIM model versus common device-independent method (Movie Mode) 
As shown in Table 7, the average visual score when using the MDCIM model at an illuminance of 1000 lux is 2.3 , i.e. between "Difference visible but reasonable" and "Difference visible, doubtful match". This is much better than the average score of 3.5 that we found for the common device-independent method for 1000 lux illuminance. On the five-point scale that we defined in Table 4, the MDCIM model leads to an average improvement of more than one unit over the common device-independent method, even at the illuminance values of 600 and 1000 lux at which the common device-independent method is relatively most accurate according to Table 7. It is not surprising that we find the relatively best color reproduction accuracy at 600 and 1000 lux ambient illuminance, because the reference conditions that were used in the derivation of sRGB color space specify even less bright ambient lighting.

For increased levels of illuminance, the average visual score for the MDCIM model remains fairly constant, with a value for 2.2 at 1500 lux and 2.6 at 3000 lux. This confirms that the MDCIM model is able to account for the effect of changing illuminance of ambient lighting, at least as long as colors do not become out-ofgamut. Table 7 shows that with the common device-independent method, observers find the quality of the color representation to become much worse at 1500 and 3000 lux.

For many samples in the test, the MDCIM model leads to a major improvement in visual score. This can be quantified by calculating the percentage of samples for which the average visual score is smaller than 3.0, i.e. for which the color match between displayed image and physical sample is found to be at least reasonable. At an illuminance of 1000 lux, this percentage is $80 \%$ for the MDCIM model, but only $20 \%$ for the common device-independent method. At 600, 1500 and 3000 lux the percentage stays fairly constant at $77 \%, 76 \%$ and $81 \%$, respectively, for the MDCIM model. For the common device-independent method the corresponding percentages are $14 \%, 6 \%$ and $8 \%$, respectively.

Figure 6 shows the distributions of visual scores for the common device-independent method and for the MDCIM model. These distributions make clear that the trends in average visual scores mentioned in the previous sections are supported by these distributions. The remaining variation in assessments from different observers may be partially due to observer metamerism, caused by the narrowband character of OLED and LCD primaries ${ }^{40}$. We conclude that for a majority of the samples the MDCIM model leads to a substantial improvement in color accuracy over the common device-independent method.

(Figure 6) 
We have searched for trends in average visual score for the various color categories mentioned in Table 5. For the common device-independent method, we found that all categories led to very similar average color scores. But for the MDCIM model, we did find differences between color categories. At all illuminance levels, the four orange colors led to visual scores much worse than other color categories. For example, at 600 lux the average visual score for orange colors is 4.1 , whereas these scores are 1.9 for yellow and for red colors, 3.1 for purple colors, 2.5 for blue colors, and 2.1 for green and for achromatic colors. Similar trends are found at larger illuminance values.

The relatively bad visual scores for the four orange colors RAL 2000, 2001, 2004 and 2008 may have two different causes. In the first place, with the MDCIM model all four orange colors already require gamut clipping at 600 lux, in order to avoid out-of-gamut colors. This is not the case for any other color category. The other cause may or may not be related to this. A visual examination of all RAL samples in a light booth under UV lighting showed that from the 35 RAL samples included in the visual test, six show fluorescence, caused by optical brighteners in the paper substrate. From these six samples, two are orange: RAL 2004 and RAL 2008. The reflection measurements that we used for generating images are based on the BYK-mac instrument, and may have been influenced by fluorescence. This was verified by comparing the reflection data with those from a different instrument (Datacolor SF600, diffuse sphere) which has a built-in UV-filter. We found that from the six fluorescent RAL samples, four indeed showed a relatively large difference in reflection data from the two instruments $\left(\Delta E_{C M C}>2.0\right.$, with $\left.l=1.5, c=1\right)$. These are the two orange samples RAL 2004 and RAL 2008, and also the purple sample RAL 4005 and the achromatic sample RAL 7021. From these four samples, we already saw that the two orange colors gave relatively visual scores in visual tests, and this is also the case for RAL 4005.

\section{4e. Results for Standard Mode (Movie Mode)}

When the Standard Mode is used for the Samsung Galaxy S4 display, the gamut of the display is extended as illustrated in Figure 1. The extension is expected to be noticeable especially for saturated green and greenish blue colors. From the samples investigated in the present study, RAL 6026 and 6029 fall into this category. These two colors are out-of-gamut when using the common device-independent method, and also when using 
the Movie Mode for 600 lux illuminance. But when using the MDCIM model for the Standard Mode, the $R$, $G$ and $B$ values for these colors reach the range between 0 and 255 (or very close to it, for RAL 6026). As an example, for RAL 6029 the $(R, G, B)$ representation with the common device-independent method is $(-90,108,56)$, placing it out-of-gamut. For 600 lux illuminance the MDCIM model representation in Movie Mode is $(-40,95,53)$ and in Standard Mode it is $(27,89,51)$. This is a first indication that the MDCIM model is capable of handling and exploiting the different color gamuts that can be realized when using displays with various specifications.

According to the results shown in Table 6, the visual tests for the device in Standard Mode show similar results as in Movie Mode. At an illuminance of 1000 lux, the MDCIM model is preferred in $70 \%$ of the assessments. At larger illuminance values, this percentage increases and reaches values well above $90 \%$ for 600 lux and 3000 lux conditions.

Table 7 shows that the common device-independent method produces slightly better visual scores for the Standard Mode than for the Movie Mode at 1000 and 1500 lux. However, for all illuminance levels the MDCIM model shows much better visual scores than the common device-independent method. The improvement varies from 0.7 units on the five-point visual scale for 1000 lux, to 1.8 units for 3000 lux. For all illuminance values, the average visual score for the MDCIM model rates between "Difference visible but reasonable" and "Difference visible, doubtful match". For the common device-independent method the average score ranks between "Difference visible, doubtful match" and "Difference clearly visible, not correct match" for 600, 1000 and 1500 lux. It even reaches between "Difference clearly visible, not correct match" and "Large difference, very bad match" for 3000 lux.

In Table 6 we found that for 3000 lux illuminance there is a very large preference (larger than $90 \%$ ) for colors produced with the MDCIM model over those from the common device-independent method. With the results presented in Table 7 this can be explained by the strong deterioration in color representation for the common device-independent method, combined with the relatively stable accuracy of color representation when using the MDCIM model.

The average visual scores shown in Table 7 can also be illustrated by calculating the percentage of samples for which the average visual score is smaller than 3, i.e. for which the color representation was assessed to be at least reasonable. At 1000 lux illuminance, for the Standard Mode when using the MDCIM model this percentage is $74 \%$, whereas for the common device-independent method it is $43 \%$. At 600,1500 and 3000 
lux this percentage becomes $86 \%, 85 \%$ and $57 \%$ for the MDCIM model, respectively. For the common device-independent method the percentages are $9 \%, 34 \%$ and $0 \%$, respectively. We conclude that also for the Standard Mode, the MDCIM model results in a substantial improvement in the accuracy of color representation for a large majority of samples.

\section{CONCLUSIONS}

We recently developed the MDCIM model (Mobile Display Characterization and Illumination Model), aiming specifically at improving the color reproduction accuracy of mobile displays. The derivation of this model accounts for the fact that mobile displays vary in technical specifications, and are used under various ambient illuminance levels.

In a previous article the MDCIM model was shown to strongly improve color reproduction accuracy of a typical mobile LCD display, as represented ${ }^{4}$ by the iPad Air 2. In the present article we investigate the performance of the model for a typical mobile OLED display, from the Samsung Galaxy S4 smartphone. With visual tests we investigated if the MDCIM model is able to produce a color representation that is more accurate than when using the common device-independent method, when compared to the color of physical RAL samples. This was tested under ambient illuminance levels varying from 600 to 3000 lux. The parameters of the model were determined based on technical data that is publicly available for the display. No additional measurement data was used.

The results of the visual tests at 600 and 1000 lux show that the common device-independent method produces an average color representation that is not very good, between "Difference visible, doubtful match" and "Difference clearly visible, not correct match". For increasing ambient illuminance levels, the color accuracy obtained with the common device-independent method becomes increasingly worse. For a vast majority of the samples, the color match becomes incorrect.

With the recently developed MDCIM model, the quality of the color match is assessed to be much better. This was found to be the case for 1000 lux ambient lighting, and even more so for lower and higher ambient illuminance levels. Our results confirm that the MDCIM model accounts not only for a wide variety of ambient illuminance levels, but also for varying technical specifications of the mobile display. 
We conclude that the MDCIM model provides a more accurate mathematical method to predict and control the color performance of mobile displays. Therefore, the MDCIM model may find application in improved color management systems on mobile devices, by providing accurate and consistent image output.

In combination with the improvements in color reproduction accuracy that we also found for LCD-based displays ${ }^{4}$, we recommend using the MDCIM model. The results obtained in the present work indicate that this requires using technical specifications of displays that can be found in public sources. For example, Refs.19, 21 provide all data required for the MDCIM model specifically for several recent OLED-based mobile displays. Examples are the displays of the Huawei Mate S, the Samsung Galaxy S6 and S7, the Samsung Galaxy Tab S2 (9.7 inch) and the HP Spectre X360. Also for many recent LCD-based mobile displays the same sources provide the required data, such as for the Microsoft Surface 3, the LG G5, the Apple iPhone 7, iPad Pro and iPad mini 4. For all these displays, the equations contained in the present article are sufficient to calculate the device-specific MDCIM model parameters. Based on the results presented, these models are expected to bring substantial improvement in color reproduction accuracy for all these devices. Therefore using the MDCIM is expected to benefit not only manufacturers of mobile OLED displays, smartphones and tablet computers, but also for example students conducting psychophysical tests using such displays.

\section{RECOMMENDATIONS FOR FUTURE WORK}

As shown in the present article and in Ref.4, the current MDCIM model results in good improvement in color reproduction accuracy for mobile displays. Manufacturers of smartphones and tablet computers may implement the model, also as part of the auto-brightness functionality that is available in most devices. Current auto-brightness functionality is known to not work satisfactory ${ }^{41}$.

In order to further develop the MDCIM model for both OLED and LCD-based displays, it may be beneficial to repeat the current tests with more observers, and to test other fit functions to represent measurement data of Tone Rendering Curves in the MDCIM model than the simple fit functions tested in the present work ${ }^{27,31}$. The common device-independent method and the MDCIM model both assume that the output of each of the primaries is independent from the degree to which the other primaries are active. But channel interaction and chromaticity shifts of the primaries may be significant for OLED displays ${ }^{27,42}$. Therefore it may be beneficial 
to investigate if these effects can be accounted for by using the Masking Model or the Modified Masking $\operatorname{Model}^{43,44}$. Further improvements may also be achieved by including a black offset in the parametrization models, and corrections for flare ${ }^{31,43,2}$.

\section{APPENDIX}

We will show that the numerical values for the gain and offset parameters used in sRGB colour space can be derived from purely mathematical and pragmatic arguments, without considering actual technical specifications of a display.

When $R G B$ values approach zero, $d_{R}, d_{G}$ and $d_{B}$ become zero as well, but according to equation (2a) the resulting luminance of the channels would only approach zero as well if the offset parameters $k_{2}$ would be chosen to be zero. Since this would seriously affect the flexibility of the model, we need an alternative solution (as for example, in Rec.709) in which the exponential form of the Tone Rendering Curves as expressed in equation (2a) is only assumed to be valid for values of $d_{R}, d_{G}$ and $d_{B}$ exceeding a threshold value $d_{0}$. For values of $d_{R}, d_{G}$ and $d_{B}$ below this threshold, a linear approximation is assumed and used in Rec.709 as in equation $(2 b)$ :

$Y_{R}=\beta \cdot d_{R}$ if $d_{R} \leq d_{0}$

Similar expressions are found for the green and blue channel. The parameter $\beta$ is a linear coefficient for which we will determine the numerical value below.

The linear interpolation of luminance $Y$ between $d=0$ and $d=d_{0}$ has the additional advantage that the Tone Rendering Curve becomes a function of $d$ that has an inverse for integer $R G B$ values. This is an important advantage in applications where the inverse of equation (1) is needed, i.e. calculating $R G B$ values from colorimetric parameters $X Y Z$ or $C I E L A B$ values.

Further, by demanding that for a white pixel with $R=G=B=255$ (i.e., for $d_{R}=d_{G}=d_{B}=1$ ) one should find a total luminance $Y=Y_{R, \max }+Y_{G, \max }+Y_{B, \max }$, it follows that:

$k_{2}=1-k_{1}$

The parts of the Tone Rendering Curve below and above the threshold are connected smoothly only if their function values and the derivatives of these functions are continuous at the threshold value. The functions in equations (2) and (A1) are continuous if 
$\left(k_{1} d_{0}+k_{2}\right)^{\gamma}=\beta d_{0}$

whereas their derivatives are continuous only if

$k_{1} \gamma\left(k_{1} d_{0}+k_{2}\right)^{\gamma-1}=\beta$

By substituting equation (A2) into (A3) and (A4) it is directly found that

$k_{1}=\frac{1}{1+(\gamma-1) d_{0}}$

$k_{2}=\frac{(\gamma-1) d_{0}}{1+(\gamma-1) d_{0}}$

$\beta=\frac{1}{d_{0}}\left(\frac{\gamma d_{0}}{1+(\gamma-1) d_{0}}\right)^{\gamma}$

The value for the threshold $d_{0}$ is put at an $R G B$ value of 10 (assuming $3 \times 8$ bits color depth). Then $d_{0}=10 / 255$

$=0.0392$. The value of gamma $\gamma$ was fixed at 2.4, for reasons described in section 2a. With these values for $d_{0}$ and $\gamma$, equations (A5), (A6) and (A7) directly lead to numerical values for the gain parameter $k_{I}=1 / 1.055$, for the offset parameter $k_{2}=0.055 / 1.055$, and for the linear coefficient $\beta=1 / 12.92$.

The numerical values for all the parameters in the SRGB color space are indeed the values found in the definition of this space ${ }^{8}$. This proves that these parameter values are not based on an analysis of technical properties of displays, but on mathematical and pragmatic arguments. 
10 


\section{REFERENCES}

1. Silverstein LD. Color display technology: from pixels to perception. IS\&T the Reporter 2006;21:1-5.

2. Cummings W and Fiske TG. Simplified Ambient Performance Assessment for Mobile Displays Using Easy Measurements. SID Digest 2014;45:528-531.

3. CIE International Commission on Illumination, Specification of colour appearance for reflective media and self-luminous display comparisons (CIE 195:2011).

4. Kirchner EJJ, van der Lans IBN, Martínez-Verdú FM and Perales E. Improving color reproduction accuracy of LCD-based mobile displays. J Opt Soc Am A 2017 (accepted).

5. ISO 14861:2015, Graphic technology -- Requirements for colour soft proofing systems.

6. Berns RS, Motta RJ, Gorzynski ME. CRT colorimetry, Part I: Theory and practice. Col Res Appl 1993;18:299-314.

7. Berns RS. Methods for characterizing CRT displays. Displays 1996;16:173-182.

8. International Electrotechnical Commission, Technical Committee 100, Audio, video and multimedia systems and equipment, Project team 61966: Color Measurement and Management in Multimedia Systems and Equipment, Part 2.1: Default RGB colour space-sRGB”, IEC 1998.

9. Kirchner EJJ, van der Lans IBN and Njo SL. Perception-based Accurate Colour Calibration of Electronic Displays. Proceedings of the 12th International Conference of the AIC (Newcastle, 2013), p. 1077-1080.

10. Berns RS, Billmeyer and Saltzman's Principles of color technology. (Wiley, third edition, New York, 2000).

11. Poynton C, Digital video and HDTV algorithms and interfaces. (Morgan Kaufmann Publishers, Amsterdam, 2003 ), p. 267.

12. International Color Consortium (ICC), online. Retrieved from http://www.color.org/srgb.xalter

13. Fairchild MD, Wyble DR. Colorimetric characterization of the Apple Studio Display (Flat Panel LCD). Munsell Color Science Laboratory Technical Report 1998.

14. Day EA, Taplin L and Berns RS. Colorimetric characterization of a computer-controlled liquid crystal display. Col Res Appl 2004;29:365-373.

15. Gao X, Khodamoradi E, Guo L, Yang X, Tang S, Guo W and Wang Y. Evaluation of colour appearances displaying on smartphones. Proceedings of the Midterm meeting of the Int'1 Colour Assn. (AIC, Tokyo, 2015), pp. 539-544.

16. Park YK, Li CJ, Luo MR, Kwak Y, Park DS and Kim C. Applying CIECAM02 for mobile display viewing conditions. Proceedings IS\&T/SID 15th Color Imaging Conference (IS\&T, Springfield, VA, 2007) 169-173.

17. Park YK, Luo MR, Li CJ and Kwak Y. Refined CIECAM02 for bright surround conditions. Col Res Appl 2015;40:114-124.

18. Ryer A, Light measurement handbook. (Technical Publications Dept., 1998).

19. Displaymate, online: www.displaymate.com

20. Anandtech, online: www.anandtech.com

21. Notebookcheck, online: www.notebookcheck.net

22. CNet, online: www.cnet.com

23. CNet data, online: http://www.cnet.com/pictures/screens-test-htc-one-vs-samsung-galaxy-s4-pictures/5/

24. Displaymate data, online: http://www.displaymate.com/Galaxy_S4_ShootOut_1.htm

25. CNet data, online: http://www.cnet.com/pictures/screens-test-htc-one-vs-samsung-galaxy-s4-pictures/7/

26. Gong R, Xu H, Wang B and Luo MR. Image quality evaluation for smartphone displays at lighting levels of indoor and outdoor conditions. Opt Eng 2012;51:1-6. 
27. Gong R, Xu H and Tong Q. Colorimetric characterization models based on colorimetric characteristics evaluation for active matrix organic light emitting diode panels. Appl Opt 2012;51:7255-7261.

28. Park TY, Jang JW, Shin KH, Yoo JJ and Shin HH. Evaluation of the ability to reproduce preferred colors for mobile displays under ambient illumination condition. SID Digest 2011;42:1253-1256.

29. Displaymate data, online: http://www.displaymate.com/Gamma_12.html

30. Kwak Y, Li C and MacDonald L. Controling color of liquid-crystal displays. Journal of the SID 2003;11:341-348.

31. Thomas JB, Hardeberg JY, Foucherot I and Gouton P. The PLVC display characterization model revisited. Col Res Appl 2008;33:449-460.

32. Bodrogi P, Sinka B, Borbély A, Geiger N and Schanda J. On the use of the sRGB colour space: the Gamma problem. Displays 2002;23: 165-170.

33. Sun PL and Luo RM. Color characterization models for OLED displays. SID Symposium Digest 2013;44:1453-1456.

34. Kirchner EJJ, van den Kieboom GJ, Njo SL, Supèr R and Gottenbos R. The Appearance of Metallic and Pearlescent Materials. Col Res Appl 2007;32:256-266.

35. Gall L. Das RAL Design System-ein neuer Farbenatlas nach CIELab, Farbe Lack. 1992;11:863-869.

36. RAL Classic System, information online: http://www.ral.de/en/ral_farben/farbkarten/ral_design.php.

37. Kang BH, Cho MS, Morovic J, Luo MR. Gamut compression algorithm development on the basis of observer experimental data. Proceedings of the IS\&T/SID Eighth Imaging Conference, 2000: 268-272.

38. Morovic J, Luo MR. The fundamentals of gamut mapping. J Imag Sci Techn 2001;45:283-290.

39. Fedorovskaya EA, de Ridder H and Blommaert FJJ. Chroma Variations and Perceived Quality of Color Images of Natural Scenes. Col Res Appl 1997;22:96-110.

40. Urban P, Fedutina M and Lissner I. Analyzing small suprathreshold differences of LCD-generated colors. J Opt Soc Am A $2011 ; 28: 1500-1512$.

41. Soneira RM. Brightness Gate for the iPhone and Android Smartphones and HDTVs - why existing brightness controls and light sensors are effectively useless. Online: http://www.displaymate.com/AutoBrightness_Controls_2.htm

42. Bastani B, Cressman B and Funt B. Calibrated color mapping between LCD and CRT Displays: a case study. Col Res Appl $2005 ; 30: 438-447$.

43. Colantoni P, Thomas JB and Hardeberg JY. High-end colorimetric display characterization using an adaptive training set. Journal of the SID 2011;19:520-530.

44. Tamura N, Tsumura N and Miyake Y. Masking model for accurate colorimetric characterization of LCD. Journal of the SID 2003;11:333-339. 

Visual scores and descriptions as used in the visual test.

Table V. Selection of RAL colors used in the visual experiment.

Table VI.

Preference of observers for the MDCIM method over the conventional sRGB method, with the Samsung S4 device either in Movie Mode or Standard Mode.

Table VII.

Average visual score obtained with the Samsung S4 device, either in Movie Mode or Standard Mode, and when using either the conventional sRGB or the proposed MDCIM method.

specifications assumed when sRGB encoding color space was defined. These specifications include the chromaticity coordinates $(x, y)$ and luminance maximum $Y_{\max }$ for the red, green and blue channel.

Display specifications for the Samsung Galaxy S4 smartphone, in Movie Mode (see main text), for chromaticity coordinates $(x, y)$ and luminance maximum $Y_{\max }$ for the red, green and blue channel [23].

Display specifications for the Samsung Galaxy S4 smartphone, in Standard Mode (see main text), for chromaticity coordinates $(x, y)$ and luminance maximum $Y_{\max }$ for the red, green and blue channel [25]. 
Table I.

\begin{tabular}{|l|l|l|l|}
\hline sRGB & Red & Green & Blue \\
\hline$x$ & 0.64 & 0.30 & 0.15 \\
\hline$y$ & 0.33 & 0.60 & 0.06 \\
\hline$Y_{\max }\left(\mathrm{cd} / \mathrm{m}^{2}\right)$ & 21.26 & 71.52 & 7.22 \\
\hline
\end{tabular}

Table II.

Table III.

Table IV.

Display specifications assumed when sRGB encoding color space was defined. These specifications include the chromaticity coordinates $(x, y)$ and luminance maximum $Y_{\max }$ for the red, green and blue channel.

\begin{tabular}{|l|l|l|l|}
\hline $\begin{array}{l}\text { Samsung Galaxy S4, Red } \\
\text { Movie Mode }\end{array}$ & Green & Blue \\
\hline $\boldsymbol{x}$ & 0.5685 & 0.2768 & 0.1706 \\
\hline$y$ & 0.3398 & 0.6167 & 0.093 \\
\hline$Y_{\max }\left(\mathrm{cd} / \mathrm{m}^{2}\right)$ & 79.392 & 217.92 & 36.739 \\
\hline
\end{tabular}

Display specifications for the Samsung Galaxy S4 smartphone, in Movie Mode (see main text), for chromaticity coordinates $(x, y)$ and luminance maximum $Y_{\max }$ for the red, green and blue channel [23].

\begin{tabular}{|l|l|l|l|}
\hline $\begin{array}{l}\text { Samsung Galaxy S4, Red } \\
\text { Standard Mode }\end{array}$ & Green & Blue \\
\hline $\boldsymbol{x}$ & 0.5774 & 0.2405 & 0.1544 \\
\hline$y$ & 0.3312 & 0.680 & 0.064 \\
\hline$Y_{\max }\left(\mathrm{cd} / \mathrm{m}^{2}\right)$ & 100.84 & 236.79 & 28.249 \\
\hline
\end{tabular}

Display specifications for the Samsung Galaxy S4 smartphone, in Standard Mode (see main text), for chromaticity coordinates $(x, y)$ and luminance maximum $Y_{\max }$ for the red, green and blue channel [25].

\begin{tabular}{|l|l|}
\hline Visual score & Description \\
\hline 0 & No / hardly any difference \\
\hline 1 & Small, negligible difference \\
\hline 2 & Difference visible but still reasonable \\
\hline 3 & Difference visible, doubtful match \\
\hline 4 & Difference clearly visible; not correct match \\
\hline 5 & Large difference; very bad match \\
\hline
\end{tabular}

Visual scores and descriptions as used in the visual test. 


\begin{tabular}{|c|c|c|c|}
\hline Category & $\begin{array}{c}\text { RAL } \\
\text { number }\end{array}$ & Category & $\begin{array}{c}\text { RAL } \\
\text { number }\end{array}$ \\
\hline \multirow[t]{4}{*}{ Yellow } & 1013 & \multirow[t]{4}{*}{ Green } & 6019 \\
\hline & 1016 & & 6021 \\
\hline & 1020 & & 6026 \\
\hline & 1024 & & 6029 \\
\hline \multirow[t]{4}{*}{ Orange } & 2000 & \multirow[t]{11}{*}{ Achromatic } & 7006 \\
\hline & 2001 & & 7013 \\
\hline & 2004 & & 7021 \\
\hline & 2008 & & 7022 \\
\hline \multirow[t]{4}{*}{ Red } & 3003 & & 7023 \\
\hline & 3012 & & 7035 \\
\hline & 3015 & & 7040 \\
\hline & 3017 & & 7044 \\
\hline \multirow[t]{4}{*}{ Purple } & 4004 & & 7047 \\
\hline & 4005 & & 9010 \\
\hline & 4008 & & 9011 \\
\hline & 4009 & & \\
\hline \multirow[t]{4}{*}{ Blue } & 5002 & & \\
\hline & 5010 & & \\
\hline & 5012 & & \\
\hline & 5024 & & \\
\hline
\end{tabular}

Selection of RAL colors used in the visual experiment.

Table VI.

\begin{tabular}{|l|l|l|}
\hline & Movie Mode & Standard Mode \\
\hline $600 \operatorname{lux}$ & $83 \%$ & $91 \%$ \\
\hline $1000 \operatorname{lux}$ & $75 \%$ & $70 \%$ \\
\hline $1500 \operatorname{lux}$ & $88 \%$ & $77 \%$ \\
\hline $3000 \operatorname{lux}$ & $95 \%$ & $97 \%$ \\
\hline
\end{tabular}

Preference of observers for the MDCIM method over the conventional sRGB method, with the Samsung S4 device either in Movie Mode or Standard Mode.

Table VII.

\begin{tabular}{|l|l|l|l|l|}
\hline & \multicolumn{2}{|l|}{ Movie Mode } & \multicolumn{2}{l|}{ Standard Mode } \\
\hline & sRGB & MDCIM & sRGB & MDCIM \\
\hline 600 lux & 3.5 & 2.4 & 3.5 & 2.1 \\
\hline 1000 lux & 3.5 & 2.3 & 3.1 & 2.4 \\
\hline 1500 lux & 4.1 & 2.2 & 3.5 & 2.2 \\
\hline 3000 lux & 4.4 & 2.6 & 4.4 & 2.6 \\
\hline
\end{tabular}

Average visual score obtained with the Samsung S4 device, either in Movie Mode or Standard Mode, and when using either the conventional sRGB or the proposed MDCIM method. 


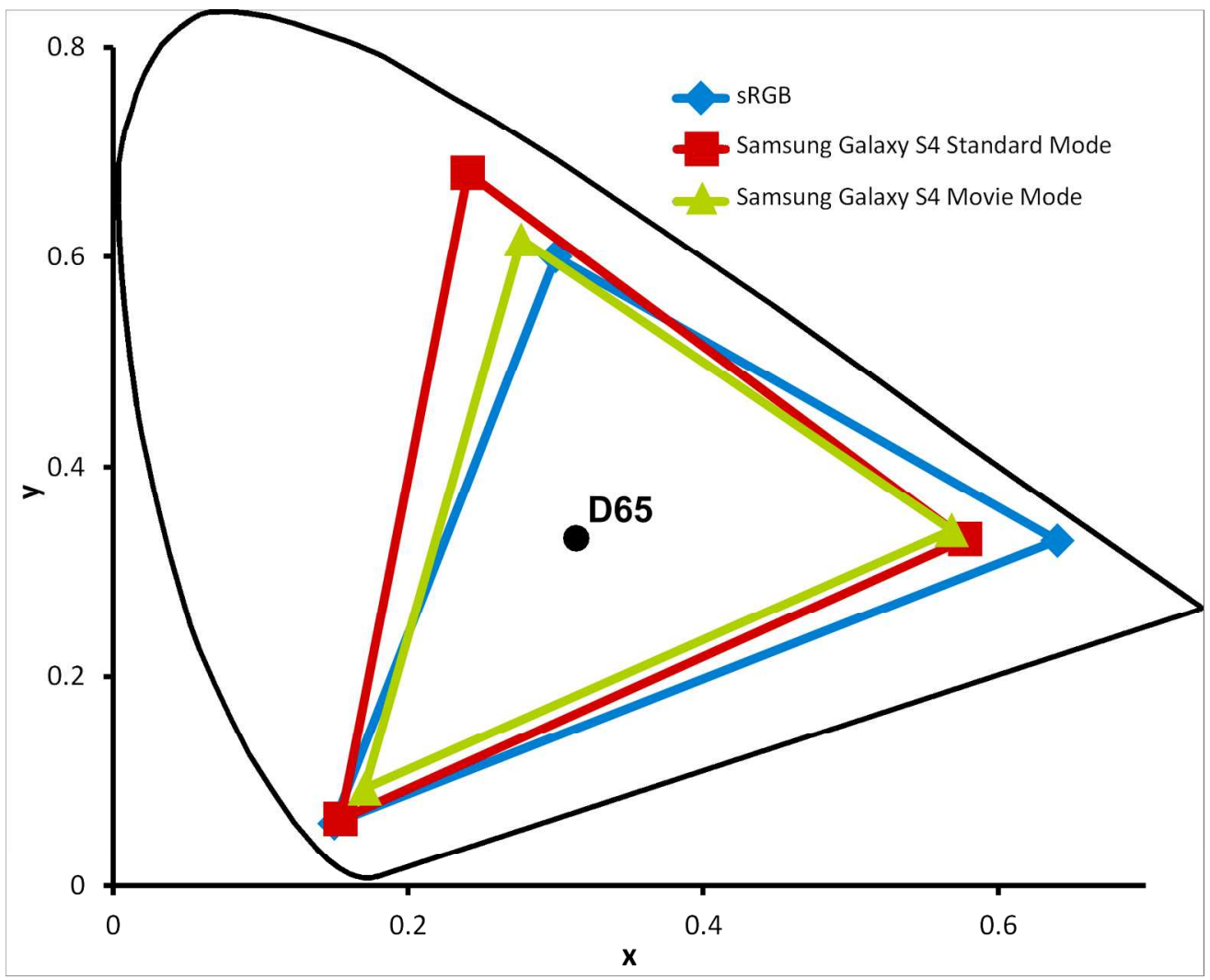

Figure 1 Color gamut from SRGB encoding color space, as compared to gamut realized with a Samsung Galaxy S4 display in Movie Mode and Standard Mode. Also the white point of D65 ambient light is shown, and the spectral loci.

$751 \times 605 \mathrm{~mm}(72 \times 72$ DPI $)$ 
1

2

3

4

5

6

7

8

9

10

11

12

13

14

15

16

17

18

19

20

21

22

23

24

25

26

27

28

29

30

31

32

33

34

35

36

37

38

39

40

41

42

43

44

45

46

47

48

49

50

51

52

53

54

55

56

57

58

59

60

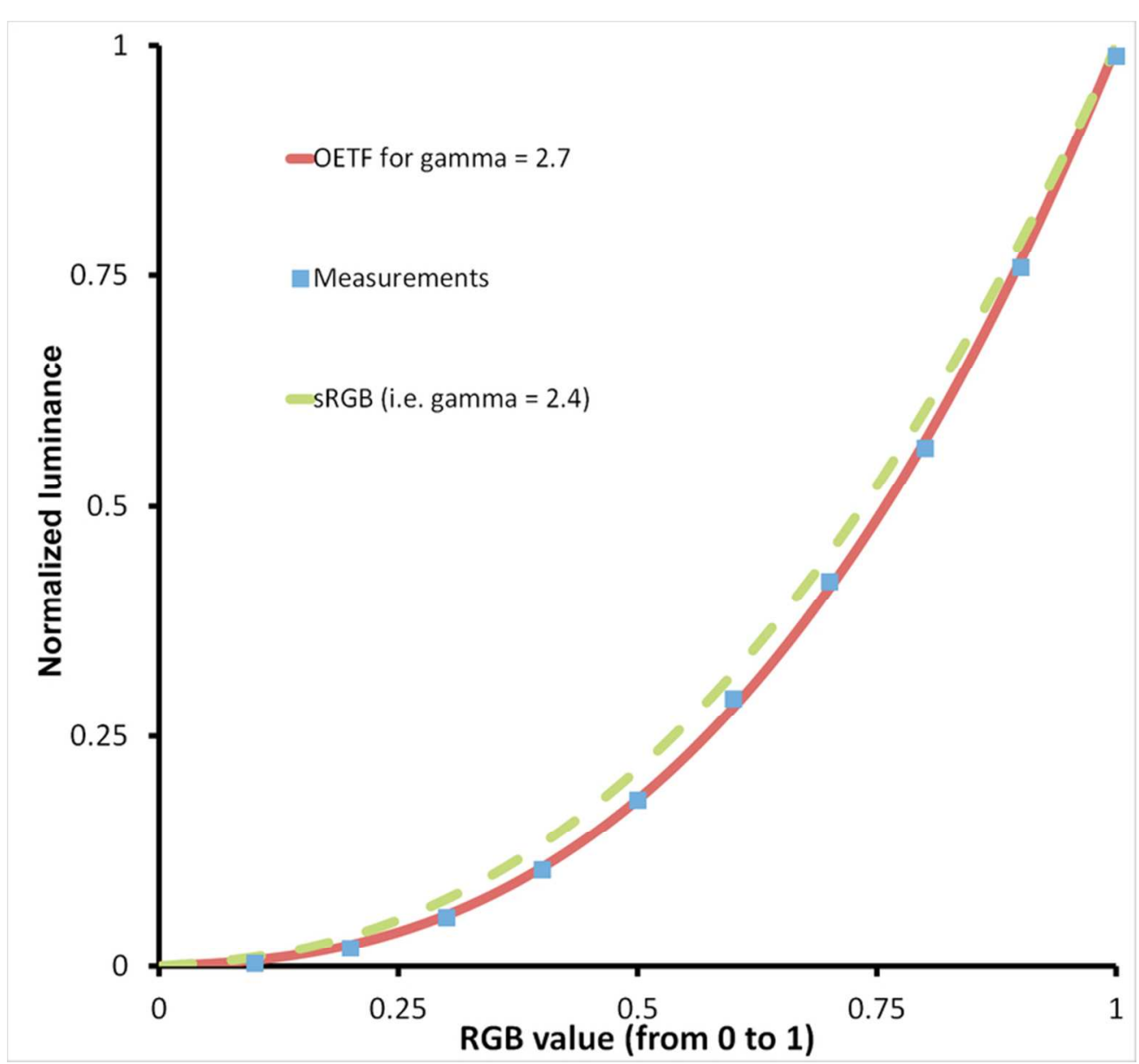

Figure 2. Tone Rendering Curve for the Samsung Galaxy S4, as measured by Displaymate [29]. The data are fitted by using $\mathrm{Y}=2.4$ and $\mathrm{Y}=2.7$.

$71 \times 65 \mathrm{~mm}(300 \times 300$ DPI $)$ 


\section{目 \\ 4G all $96 \%$ 16:53}

\section{Test_sRGB}

\section{Top color is best match}

\section{Take new paint sample}

Get sample: A10 HON5874/ A1

\section{= Nr 3 from 21 samples}

\section{Bottom color is best match}

Figure 3a Screen shots of software application used in visual test.

$$
81 \times 144 \mathrm{~mm}(300 \times 300 \text { DPI) }
$$




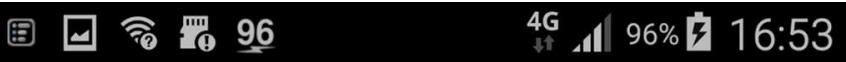 \\ Test_sRGB}

\section{Top color is best match}

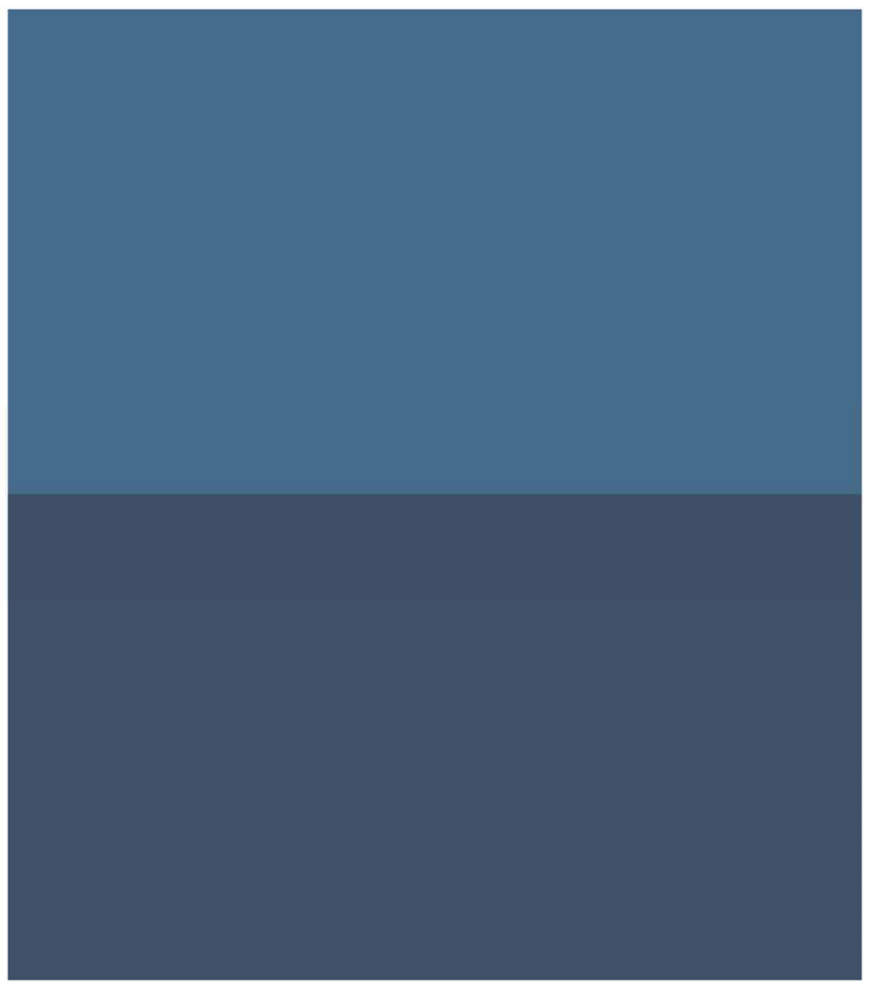

\section{Bottom color is best match}

Figure 3b Screen shots of software application used in visual test.

$81 \times 144 \mathrm{~mm}(300 \times 300$ DPI $)$ 


\section{国 $\square$ \% 罾 96 \\ 4G $96 \% 16: 54$}

\section{Test_sRGB}

Sample name: A10 HON5874/A1

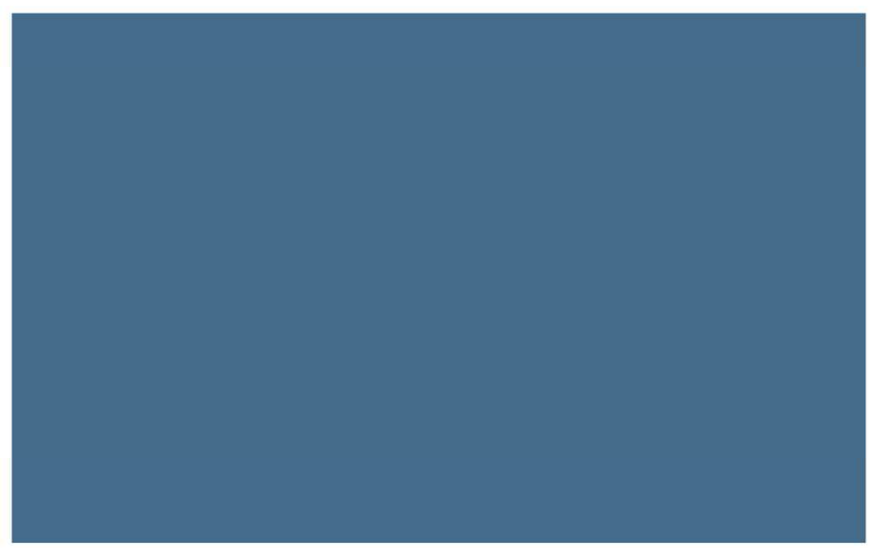

Color match score for this top color against paint sample:

Difference visible but still reasonable 2.00

\section{Continue}

Figure 3c Screen shots of software application used in visual test.

$$
81 \times 144 \mathrm{~mm}(300 \times 300 \text { DPI) }
$$




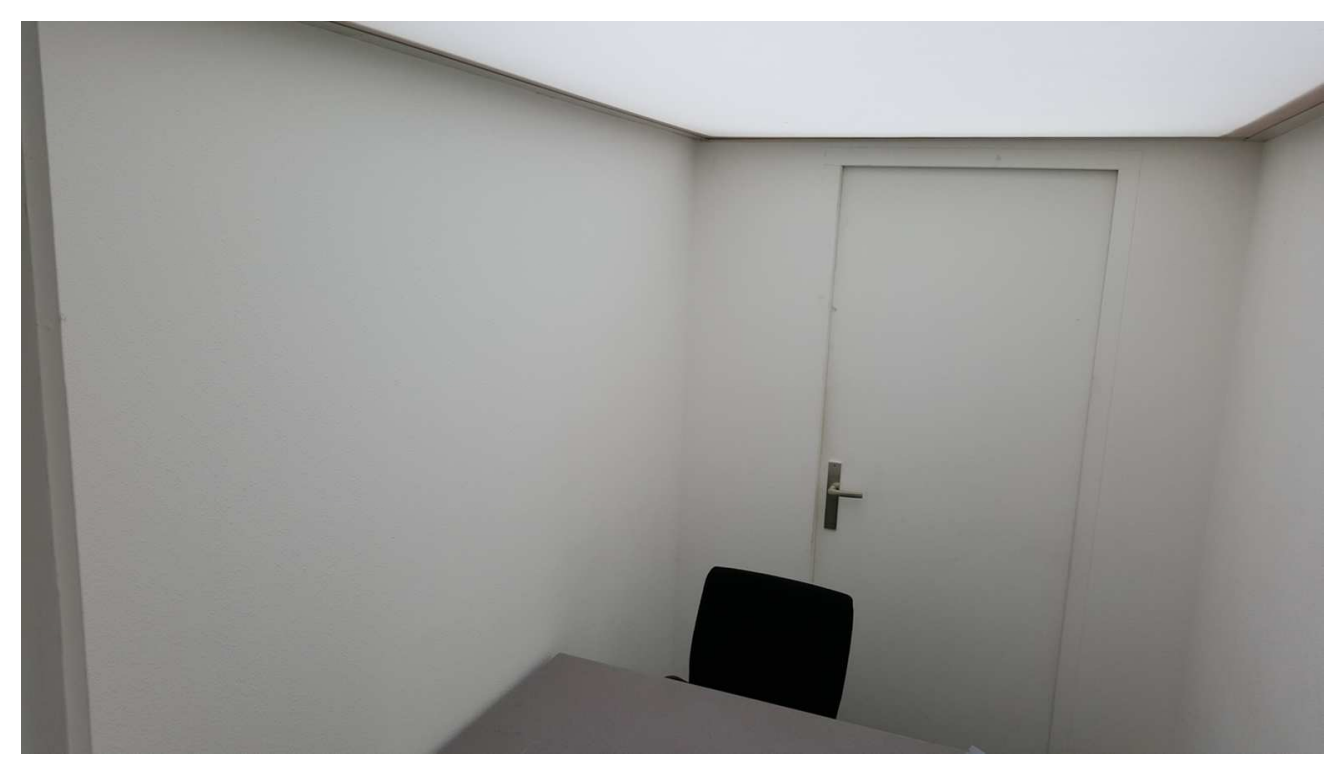

Figure 4 (a) Room with diffuse lighting as used in visual test.

$63 \times 35 \mathrm{~mm}(600 \times 600 \mathrm{DPI})$ 


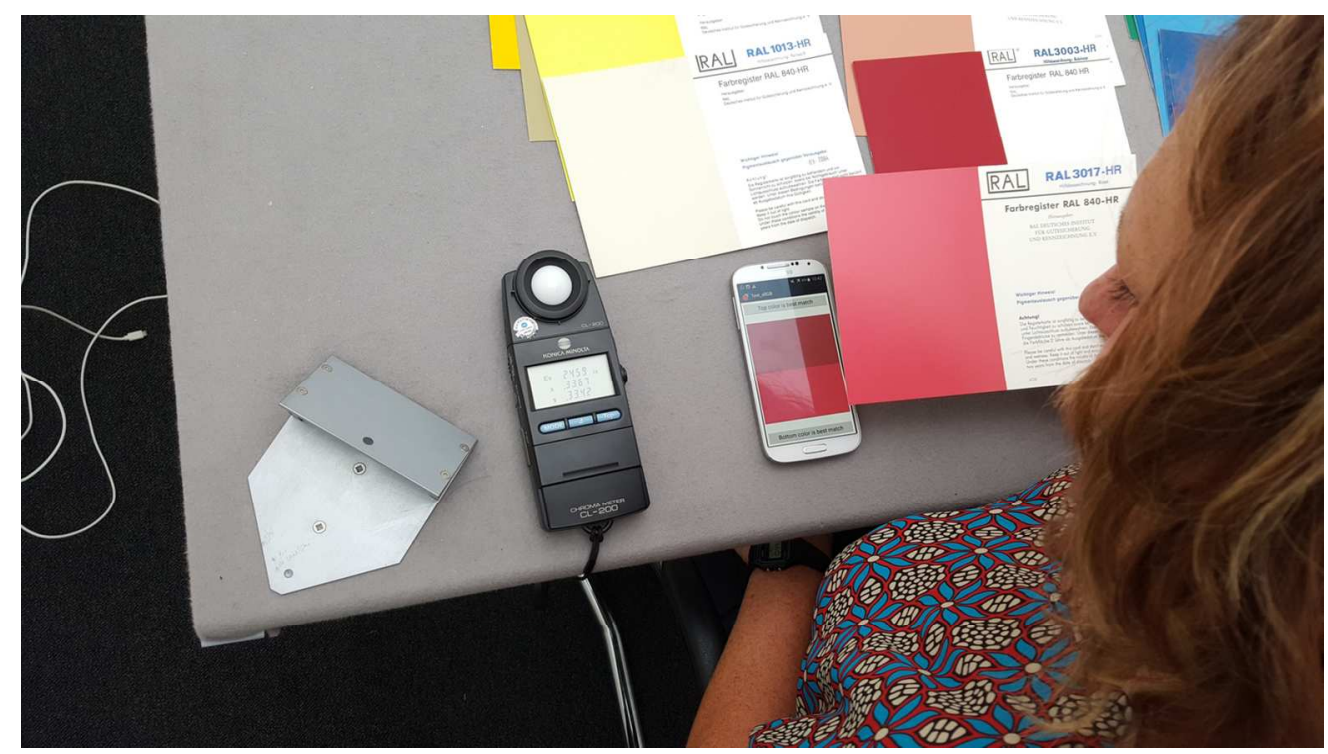

Figure 4 (b) observer assessing accuracy of color representation on mobile display.

\section{$63 \times 35 \mathrm{~mm}(600 \times 600 \mathrm{DPI})$}


1

2

3

4

5

6

7

8

9

10

11

12

13

14

15

16

17

18

19

20

21

22

23

24

25

26

27

28

29

30

31

32

33

34

35

36

37

38

39

40

41

42

43

44

45

46

47

48

49

50

51

52

53

54

55

56

57

58

59

60

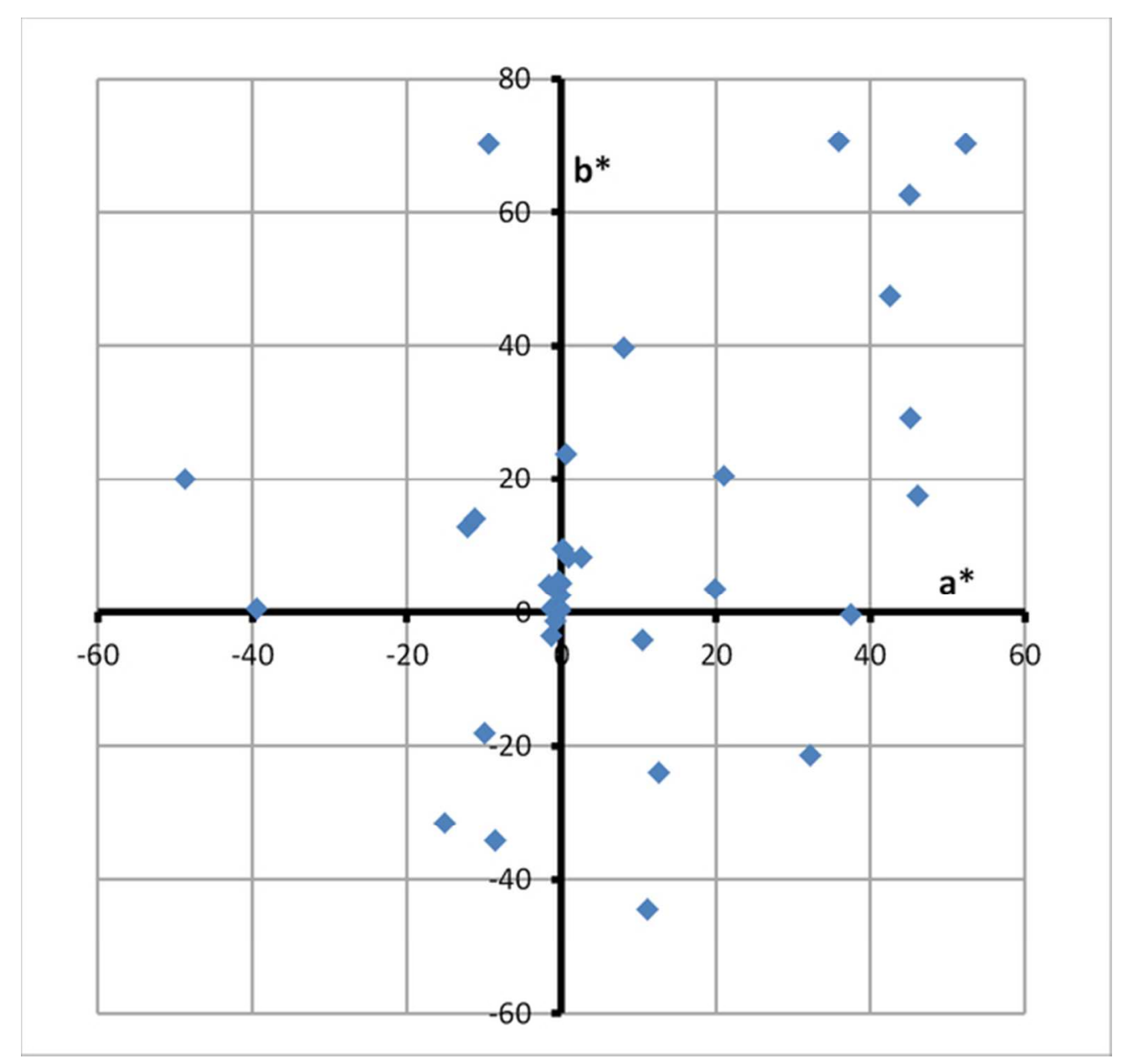

Figure 5a Distribution of colors measured on the 35 samples used in the present investigation, as distributed in the (a) CIE-a*, b* plane and in the (b) CIE-h, L* plane.

$54 \times 51 \mathrm{~mm}(300 \times 300 \mathrm{DPI})$ 
Figure $5 \mathrm{~b}$ Distribution of colors measured on the 35 samples used in the present investigation, as distributed in the (a) CIE-a*, b* plane and in the (b) CIE-h, L* plane.

$54 \times 48 m m(300 \times 300$ DPI $)$ 


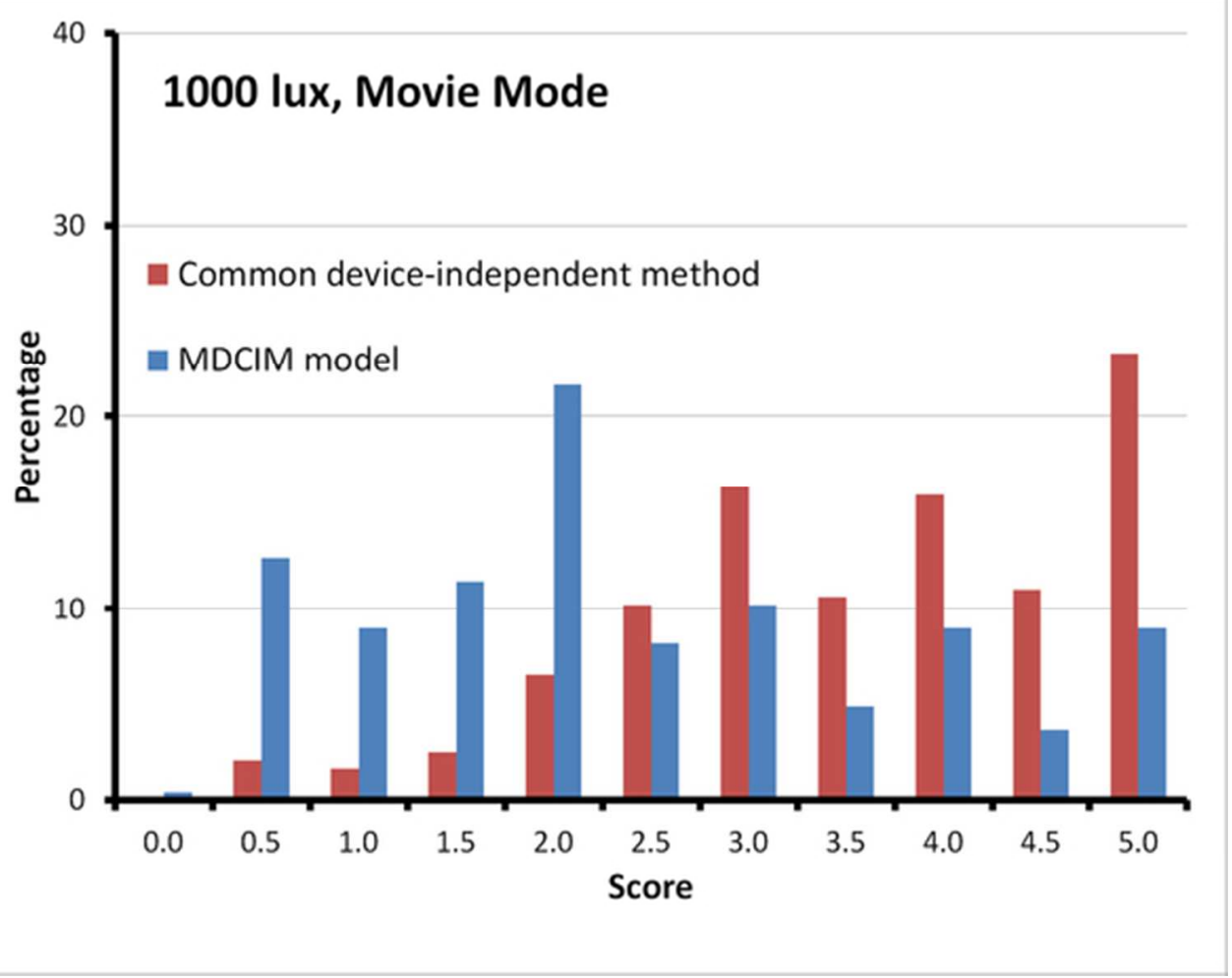

Figure 6a Distribution of visual scores for 1000 and 1500 lux, when device is in Movie Mode.

$$
51 \times 39 \mathrm{~mm}(300 \times 300 \mathrm{DPI})
$$




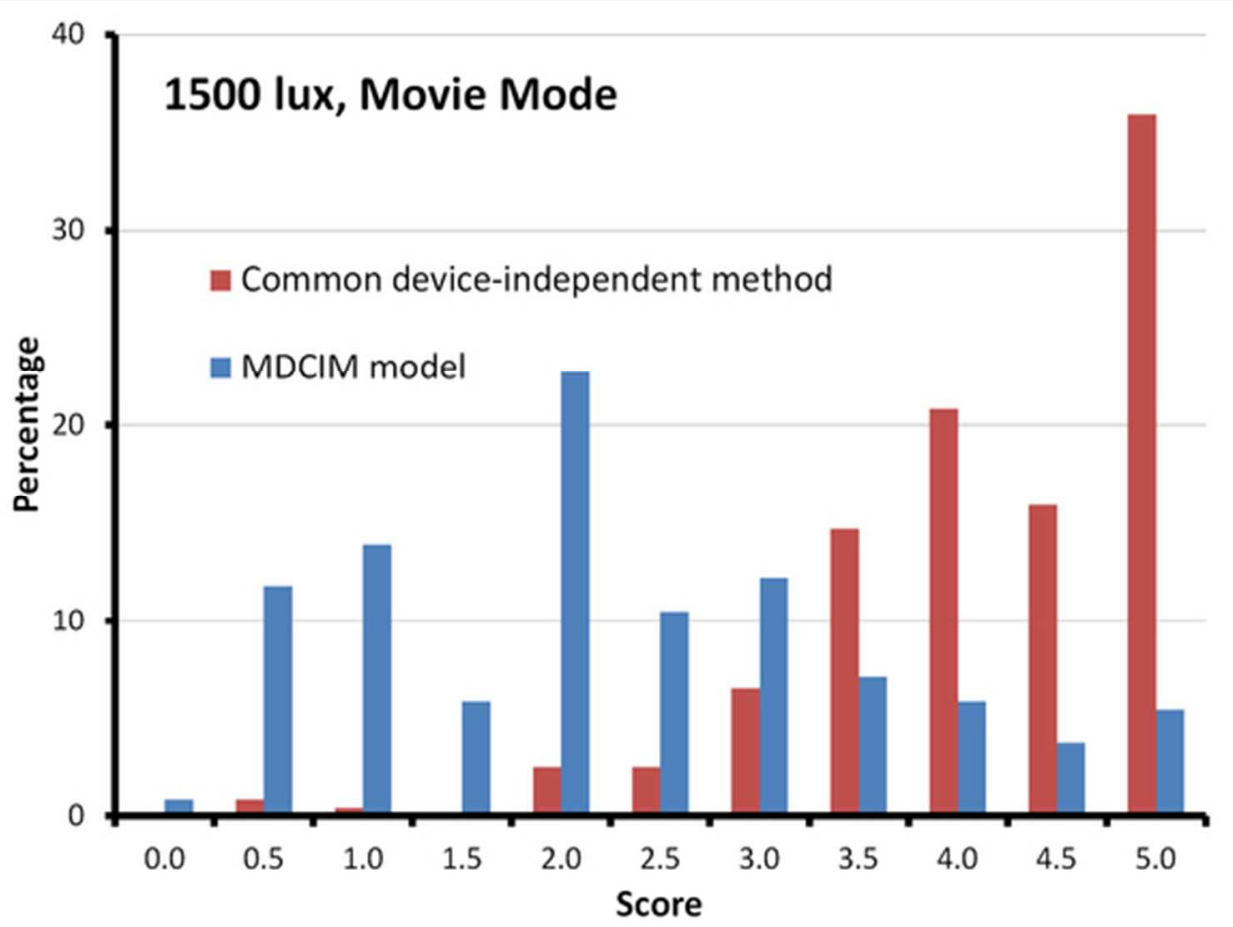

Figure 6b Distribution of visual scores for 1000 and 1500 lux, when device is in Movie Mode.

$$
51 \times 39 \mathrm{~mm}(300 \times 300 \text { DPI })
$$

